http://jmscr.igmpublication.org/home/

ISSN (e)-2347-176x ISSN (p) 2455-0450

crossref DOI: https://dx.doi.org/10.18535/jmscr/v8i1.164

Journal Of Medical Science And Clinical Research

\title{
Tuberculosis of Shoulder Joint - A Rare Presentation
}

\author{
Authors \\ Dr Zahid Hussain Thakkallapalli ${ }^{1}$, Dr B. Udaya Kumar ${ }^{2}$, \\ Dr Ch. Deepak ${ }^{3}$, Dr B. Monica Lakhmi ${ }^{4}$ \\ ${ }^{1}$ Post Graduate, Dept. of Orthopaedics, GEMS \& Hospital, Srikakulam \\ ${ }^{2}$ Head of Department, Professor, GEMS, Srikakulam \\ ${ }^{3}$ Asst. Professor, Department Of Orthopaedics, GEMS, Srikakulam \\ ${ }^{4}$ Post Graduate, Dept of Orthopaedics, GEMS Hospital, Srikakulam
}

\begin{abstract}
Tuberculosis of joint is common but involvement of shoulder joint is rare. We present a case of shoulder tuberculosis of a 43 year old male who presented with pain in left shoulder joint for last 7 months and swelling over the arm from 5 months. Patient had restricted range of movements. X-ray, CT scan showed decreased joint space and multiple lytic areas. MRI showed intra articular and peri articular collection of fluid and gave diagnosis of tuberculosis. Excision Biopsy was done by arthroscopic technique and sent for Histopathological examination and CBNAAT (cartridge based nucleic acid amplification test) which were reported as positive for tuberculosis. Patient was treated with Arthroscopic debridement and Anti tuberculosis therapy for 9 months and has regained good range movements.
\end{abstract}

\section{Introduction}

Tuberculosis is most prevalent in India and skeletal tuberculosis affects most commonly next to pulmonary tuberculosis. In skeletal tuberculosis, spine is most commonly affected followed, by hip and knee. Tuberculosis in shoulder joint is very rare and its incidence is about $1-2 \%$ of total skeletal tuberculosis ${ }^{1,2,3,4}$. Here we present a case of caseating exudata form of shoulder tuberculosis.

\section{Case Report}

A 43 year old male came with pain over left shoulder from 7 months who was apparently normal before, later he developed pain in left shoulder which was insidious in onset and gradually progressive leading to limitation of left shoulder movements affecting his day to day activities and it was dull aching pain, nonradiating, aggravated on movement of left shoulder and relieved on taking rest and NSAIDS. He also developed swelling in mid arm 2 months after onset of shoulder pain, which appeared as small swelling and gradually increased extending anterior, medial and lateral portion of arm. Patient had history of low grade fever with evening raise in temperature and increased pain during night. $\mathrm{He}$ had no history of cough and breathlessness. No family history of tuberculosis. Patient was moderately built and nourished, systemic examination is within normal limits. Local examination revealed wasting of deltoid, supraspinatus and infraspinatus muscle with fullness over supra clavicular region .A swelling 
was seen around mid arm of size 7 X $5 \mathrm{cms}$ extending medially and anterio-laterally. There was local raise of temperature around left shoulder joint and tenderness was present in both anterior and posterior gleno-humeral joint line .Arm swelling had no local raise of temperature. It was soft in consistency, fluctuation present, lower and lateral borders are well defined, superior and medial borders are diffuse .Skin was pinchable over the swelling. Swelling was mobile and Trans illumination test was negative.Palpation of clavicle, acromio-clavicular joint, spine of scapula and coracoid process are normal.There is a painful restriction of movements with flexion up to 60 degrees, extension up to 20 degrees, abduction of about 20 degrees and rotation of 20 degrees each. Apical and medical group of axillary lymph nodes were palpable along with supraclavicular nodes which were non-tender.

\section{Clinical Picture}

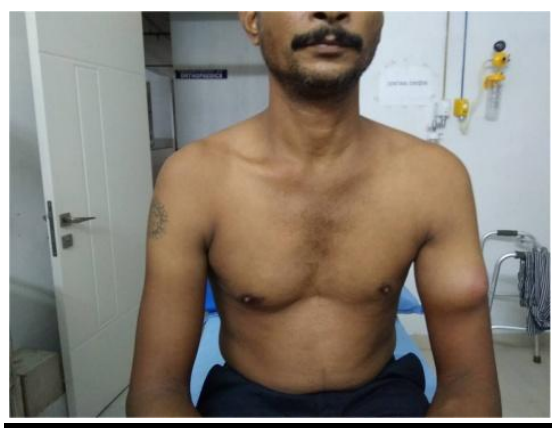

Figure-1

\section{X-Ray Findings}

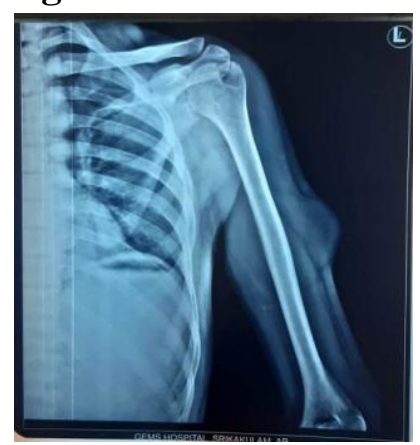

Figure -2

X-ray left shoulder AP view revealed Narrowing of joint space with irregularity of articular margin of head of humerus.there was Superior migration of head of humerus and Osteolytic lesion in greater tuberosity. Osteoporosis of humerus, acromion and outer end of clavicle was also noted.soft tissue shadow has been noted at middle third of humerus

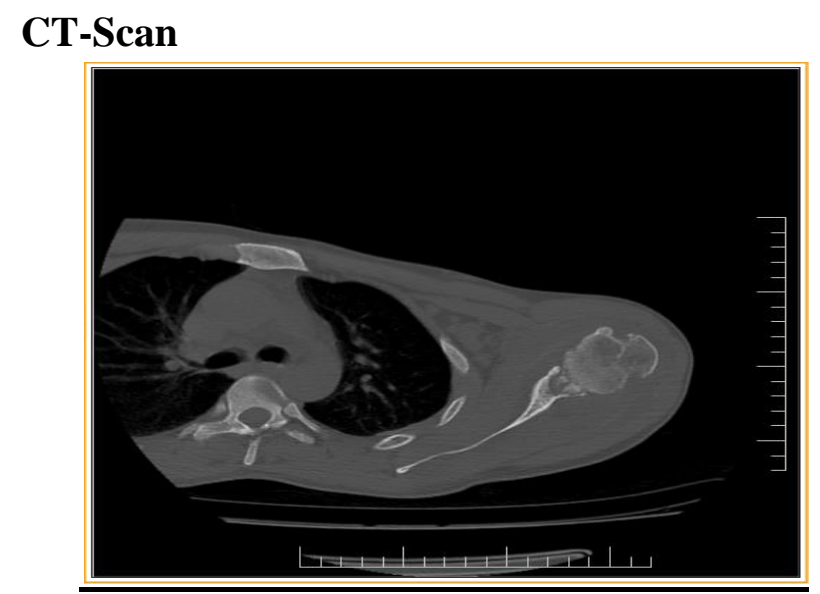

Figure-3

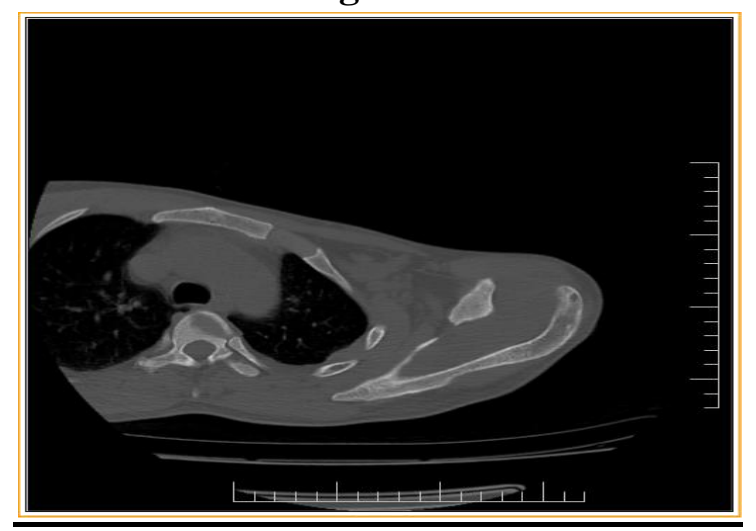

Figure-4

Ct left shoulder showed Multiple irregular lytic areas seen on greater tubercle and lesser tubercles of left humerus with irregular margins and reduced joint space,Multiple lytic areas and erosions noted in the neck of the scapula, glenoid, articular surface of head of humerus and acromion process of left shoulder joint and it also showed multiple enlarged oval to round axillary and supraclavicular lymph nodes.To know the extent of the disease in to soft tissues we have taken MRI left shoulder joint where we have found gross intra articular collection with extensive periarticular extension into intra and intermuscular plane. Erosions over humeral head, glenoid, acromion are found with intense peri-erosional edema, loose bodies were found and features were highly suggestive for tubercular arthritis rather than pyogenic. 
Treatment was arthroscopic debridement of the shoulder joint and incision drainage of cold abscess with immediate closure and streptomycin instillation in the joint and cold abscess.

Material collected was sent for histopathology and amplification test where biopsy report was granulomatous inflammation and amplification test gave positive results for tuberculosis.

Patient was immobilized for 3 days postoperatively with arm pouch and later he was encouraged for passive and active movements of the shoulder joint. He was advised for anti tuberculous drug therapy for 18 months.

Follow up was done for 9 months once in every 2 months and gradual decrease in pain was noted with increased range of movements.
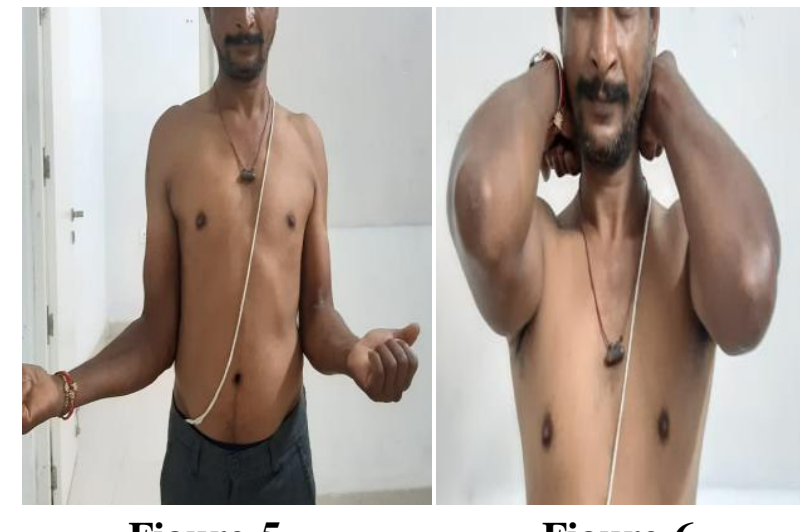

Figure-5

Figure-6

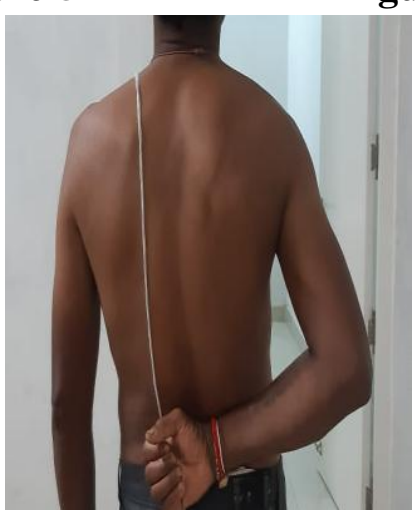

Figure-7

\section{Discussion}

Skeletal tuberculosis is rare compared to the pulmonary form. The incidence of shoulder tuberculosis is about $1-2.8 \%$ of total musculoskeletal tuberculosis ${ }^{1,2}$.

Shoulder tuberculosis is generally considered as two types (1)dry type or caries sicca (2)fulminating type also known as caries exudate is normally associated with cold abscess or sinus formation. Here, our patient has been presented with caries exudate type $\mathrm{e}^{1,3}$.

Basing on the clinical and radiological evidences we had differential diagnosis of pyogenic arthritis, rheumatoid arthritis, adhesive capsulitis and synovial neoplastic conditions ${ }^{5}$.

Though x ray, CT scan and MRI are suggestive of tuberculosis, they will not confirm diagnosis for which biopsy is gold standard ${ }^{7}$.

Treatment mainly includes anti tubercular drugs consisting of Rifampicin, Isoniazid, Streptomycin, Ethambutol, Pyrazinamid. Surgical therapy can be done basing on the clinical condition of the patient and options generally include partial synovectomy, abscess drainage and its debridement. Our patient has received Arthoscopic debridement of joint and drainage of abscess followed by anti microbial therapy for 9 months till now and patient has good range of shoulder movements.

\section{Conclusion}

Tuberculosis of shoulder joint is a rare disease entity and it should be suspected in cases of long standing joint pain with low grade fever and person from endemic areas like in India and in patients having previous history of tuberculosis. Though radiology gives good picture regarding joint destruction but it is generally difficult diagnose in early stages and by the time it is quantified radiologically, the bone and joint are generally destroyed. Therefore tuberculosis should be suspected in cases of long standing pain in the shoulder. Arthrodesis is generally reserved for failed chemotherapy cases and excessively destroyed joints and is not considered now a day. Arthroscopic debridement of the shoulder joint and early mobilization gives good results.

\section{References}

1. Tuli SM. Tuberculosis of the Skeletal System. 2nd Edition. Bangalore;. Jaypee Brothers. 1997: 6 and 123-126. 
2. Mehta MT. Prognosis in tuberculosis arthritis. In Current concepts in bone and joint tuberculosis. Ed. TK Shanmugasundaram. Madras; Madras documentation Center, International Bone and Joint Club. 1985: 78.

3. Duthie RB, Ferguson AB. Mercer's Orthopaedic Surgery. 7th Edition. London; Edward Arnold [Publishers]Ltd. 1973:559560.

4. Tang SC, Chow SP. Tuberculosis of Shoulder: Report of 5 cases treated conservatively. J Roy Coll Surg Edin 1983; 283:188-90

5. Donald Resnick, Gen Niwayama, Diagnosis of bone and joint Disorder 2nd ed. Vol.4, W.B. Saunders Publication: 2678-2685

6. Martini M. Tuberculosis of bone and joints. Springer-Verlag; Heidelberg: 1998.

7. Davis IR, Hamilton A, Biggart JD. Primary synovial chondromatosis: a clinocopathologic review and assessment of malignant potential. Hum Pathol. 1998;29:683-88. 